Journal of Applied Analysis

Vol. 6, No. 1 (2000), pp. 77-85

\title{
ON ANALOGUES OF SOME CLASSICAL SUBSETS OF THE REAL LINE
}

\author{
A.B. KHARAZISHVILI \\ Received December 17, 1998 and, in revised form, September 22, 1999
}

\begin{abstract}
A theorem on the existence of separable supports of $\sigma$-finite Borel measures given on metric spaces with small topological weights is applied to constructions of certain analogues of special subsets of the real line.
\end{abstract}

A well-known result from topological measure theory states that every $\sigma$-finite Borel measure on a metric space whose weight is not real-valued measurable possesses a separable support (cf. [7] and [10]). The main goal of the present article is to demonstrate several applications of this result to some generalized analogues of classical subsets of the real line $\mathbf{R}$ (e.g. Luzin sets, Sierpiński sets and so on).

We assume that all topological spaces $E$ under consideration below satisfy the following condition: all one-element subsets of $E$ are Borel (in $E$ ). The symbol $\omega$ denotes, as usual, the first infinite cardinal, $\omega_{1}$ - the first uncountable cardinal and $\mathbf{c}$ denotes the cardinality continuum.

We recall that $E$ is a universal measure zero space (or universally negligible space) if there is no nonzero $\sigma$-finite diffused Borel measure on $E$.

The so-called Luzin subsets of the real line $\mathbf{R}$ yield nontrivial examples of uncountable universal measure zero spaces. According to the classical

1991 Mathematics Subject Classification. Primary 28A05, 28D05.

Key words and phrases. Separable support of a measure, Luzin set, Sierpiński set, universal measure zero space.

ISSN 1425-6908 CHeldermann Verlag. 
definition, a set $X \subset \mathbf{R}$ is a Luzin set (respectively, a generalized Luzin set) if $X$ is uncountable (respectively, $\operatorname{card}(X)=\mathbf{c}$ ) and, for each first category set $Z \subset \mathbf{R}$, we have $\operatorname{card}(X \cap Z) \leq \omega$ (respectively, $\operatorname{card}(X \cap Z)<\mathbf{c})$.

It is well known (see, e.g., [6]-[10]) that the Continuum Hypothesis (respectively, Martin's Axiom) implies the existence of Luzin sets (respectively, of generalized Luzin sets). In addition, if Martin's Axiom and the negation of the Continuum Hypothesis hold, then there are no Luzin subsets of the real line. In particular, the question of the existence of Luzin sets on $\mathbf{R}$ is undecidable in the theory ZFC (for more details, see [6]).

Many years ago Luzin showed that every Luzin set on $\mathbf{R}$ is universal measure zero. This evidently implies that, under certain set-theoretical assumptions, there are uncountable universal measure zero subspaces of $\mathbf{R}$. Later on, Luzin also demonstrated in ZFC, by using some methods of the theory of analytic sets, that there exist universal measure zero subspaces of $\mathbf{R}$ having cardinality $\omega_{1}$ (see, e.g., [7] and [8]). This classical result is sharp because, as established by Baumgartner and Laver, some models of set theory are possible where the cardinality of each universal measure zero subset of $\mathbf{R}$ is less than or equal to $\omega_{1}$ (cf. [8] and the references therein). Also, as mentioned above, if Martin's Axiom holds, then there are generalized Luzin subsets of $\mathbf{R}$ being simultaneously universal measure zero spaces of cardinality continuum. On the other hand, if the cardinality continuum is real-valued measurable, then there is no universal measure zero set in $\mathbf{R}$ of cardinality $\mathbf{c}$.

It is interesting to note that, replacing $\mathbf{R}$ by some natural nonseparable spaces, we obtain an essentially different situation. The statement below illustrates this fact.

Theorem 1. Let $H$ be a Hilbert space over $\mathbf{R}$ whose Hilbert dimension is c (i.e. any orthonormal basis in $H$ has cardinality c). The following two assertions are true:

1 ) if $\mathbf{c}$ is not real-valued measurable, then there exists a universal measure zero subspace $X$ of $H$ which is everywhere dense in $H$ (in particular, $\operatorname{card}(X)=\mathbf{c})$

2 ) if $\mathbf{c}$ is a regular cardinal and the negation of the Continuum Hypothesis is fulfilled, then there exists no generalized Luzin subset of $H$, i.e. there exists no subset $X$ of $H$ with $\operatorname{card}(X)=\mathbf{c}$ such that, for any first category set $Z \subset H$, the inequality $\operatorname{card}(X \cap Z)<\mathbf{c}$ is satisfied.

Proof. Notice that, since Hilbert dimension of $H$ is $\mathbf{c}$, the cardinality of $H$ is also equal to c. Moreover, it is not hard to see that each everywhere dense subset of $H$ has cardinality c.

First, we are going to show that 1 ) is true. For every natural number $n>0$, let us denote by $\left(U_{i, n}\right)_{i \in I}$ a covering of $H$ by open balls with radius 
$1 / n$. We may suppose without loss of generality that the set $I$ is well ordered by $\preceq$ and that

$$
U_{i, n} \backslash \bigcup\left\{U_{j, n}: j \prec i\right\} \neq \emptyset
$$

for all $i \in I$ (indeed, if the latter set-theoretical difference is empty, then we can withdraw $U_{i, n}$ from our covering). Pick any point $x_{i, n}$ from $U_{i, n} \backslash$ $\bigcup\left\{U_{j, n}: j \prec i\right\}$ and put

$$
X_{n}=\left\{x_{i, n}: i \in I\right\} .
$$

It is not difficult to check that the set $X_{n}$ is $\sigma$-discrete in the space $H$ (cf. [7] or [10]) and forms a $(2 / n)$-net in $H$. Further, we put

$$
X=\bigcup\left\{X_{n}: 0<n<\omega\right\}
$$

and we assert that $X$ is the required subset of $H$. The density of $X$ in $H$ is evident. Consider now an arbitrary Borel diffused probability measure $\mu$ on $H$. Since $\mathbf{c}$ is not real-valued measurable, there exists a closed separable set $F$ in $H$ such that $\mu(F)=1$. In virtue of our construction, we may write

$$
\operatorname{card}\left(X_{n} \cap F\right) \leq \omega,
$$

for each $n>0$, because $X_{n}$ is $\sigma$-discrete and $F$ is separable. Consequently, we get $\operatorname{card}(X \cap F) \leq \omega$. This immediately implies that

$$
\mu^{*}(X \cap F)=0
$$

and hence

$$
\mu^{*}(X) \leq \mu^{*}(X \cap F)+\mu^{*}(X \cap(H \backslash F))=0
$$

which, in particular, shows that $X$ is universal measure zero.

Now, let us establish that 2) is true, too. Let the assumption of 2) be satisfied, and suppose to the contrary that there exists a generalized Luzin subset $X$ of $H$. It is not hard to check that $H$ can be represented in the form

$$
H=\bigcup\left\{V_{\xi}: \xi<\omega_{1}\right\}
$$

where all $V_{\xi}$ are proper closed vector subspaces of $H$. Indeed, $H$ is the orthogonal sum of two its Hilbert subspaces $H^{\prime}$ and $H^{\prime \prime}$ where $H^{\prime}$ has Hilbert dimension $\omega_{1}$. Let $\left\{e_{\xi}: \xi<\omega_{1}\right\}$ be an orthogonal basis of $H^{\prime}$. Then, for any ordinal $\xi<\omega_{1}$, we put $V_{\xi}=$ the Hilbert space generated by $H^{\prime \prime} \cup\left\{e_{\zeta}: \zeta<\xi\right\}$.

Evidently, each set $V_{\xi}$ is nowhere dense in $H$. Consequently,

$$
\mathbf{c}=\operatorname{card}(X)=\sum_{\xi<\omega_{1}} \operatorname{card}\left(X \cap V_{\xi}\right) .
$$

In other words, we obtain that $\mathbf{c}$ is a singular cardinal. But this is impossible.

In particular, we see that, assuming Martin's Axiom and the negation of the Continuum Hypothesis there are no generalized Luzin subsets of $H$. 
The theorem has thus been proved.

We shall say that a topological space $E$ is a Sierpiński space if $E$ does not contain a universal measure zero subspace whose cardinality is equal to $\operatorname{card}(E)$.

It immediately follows from this definition that all Sierpiński spaces are uncountable.

The classical Sierpiński subset of the real line $\mathbf{R}$ (see, e.g., [6]-[10]) may be regarded as a standard example of a Sierpiński space. The existence of such a subset of $\mathbf{R}$ can be established under the Continuum Hypothesis. If one assumes Martin's Axiom, then there exists a so-called generalized Sierpiński subset of $\mathbf{R}$ which is a Sierpiński space, too. It is well known that the existence of a classical Sierpiński set on $\mathbf{R}$ cannot be proved within the theory ZFC. For instance, if Martin's Axiom and the negation of the Continuum Hypothesis hold, then there is no Sierpiński set on $\mathbf{R}$ (see, e.g., [6]). On the other hand, it is not hard to construct uncountable Sierpiński spaces within the theory ZFC (see, e.g., the papers [4] and [5] where it is shown that, for any infinite cardinal $\omega_{\beta}$ whose cofinality is strictly greater than $\omega$, there exists a Sierpiński space $E$ with $\left.\operatorname{card}(E)=\omega_{\beta}\right)$. Some general properties of Sierpiński spaces are discussed in these papers, too. In particular, it is demonstrated there that the union of a finite family of Sierpiński subspaces of a given topological space is also a Sierpiński space; at the same time, the topological sum of a countable family of Sierpiński spaces is not, in general, a Sierpiński space. In the present article, we shall deal with some other properties of Sierpiński spaces (in this connection, let us remark that no purely topological characterization of Sierpiński spaces is known).

We begin with the following statement.

Theorem 2. Let $E$ be a Sierpiński space, let $E^{\prime}$ be a topological space with $\operatorname{card}\left(E^{\prime}\right)=\operatorname{card}(E)$, such that there exists a Borel surjection from $E$ onto $E^{\prime}$. Then $E^{\prime}$ is a Sierpiński space, too.

Proof. Let $g: E \rightarrow E^{\prime}$ be a Borel surjection. Suppose to the contrary that $E^{\prime}$ is not a Sierpiński space. Then there exists a set $Y \subseteq E^{\prime}$ with $\operatorname{card}(Y)=\operatorname{card}\left(E^{\prime}\right)$ which is a universal measure zero space. Consider the family

$$
\left\{g^{-1}(y): y \in Y\right\}
$$

of nonempty sets and denote by $X$ a selector of this family. Clearly, we have

$$
\operatorname{card}(X)=\operatorname{card}(Y)=\operatorname{card}\left(E^{\prime}\right)=\operatorname{card}(E)
$$

and the mapping $g \mid X$ is a Borel bijection $f$ between $X$ and $Y$. Since $E$ is a Sierpiński space, $X$ is not universal measure zero. Hence there exists a 
Borel diffused probability measure $\mu$ on $X$. But, starting with this measure, we can define a Borel diffused probability measure $\nu$ on $Y$ by

$$
\nu(Z)=\mu\left(f^{-1}(Z)\right)(Z \in B(Y))
$$

where $B(Y)$ denotes, as usual, the Borel $\sigma$-algebra of $Y$. We thus obtain a contradiction with our assumption that $Y$ is a universal measure zero space.

Consider the real line $\mathbf{R}$ equipped with its standard topology. The question whether $\mathbf{R}$ is a Sierpiński space cannot be decided in the theory $\mathbf{Z F C}$. Indeed, if Martin's Axiom holds, than there exists a generalized Luzin subset of $\mathbf{R}$ which is a universal measure zero space. Since the cardinality of this subset is equal to the cardinality continuum $\mathbf{c}$, we conclude that, under Martin's Axiom, $\mathbf{R}$ is not a Sierpiński space. On the other hand, suppose that the cardinality continuum is real-valued measurable and that $\mathbf{R}$ is not a Sierpiński space. Then there exists a universal measure zero subspace $Z$ of $\mathbf{R}$ whose cardinality equals $\mathbf{c}$. But this is impossible, because, taking into account the real-valued measurability of $\mathbf{c}$, we can easily define a Borel diffused probability measure on $Z$. Thus, the real-valued measurability of $\mathbf{c}$ implies that $\mathbf{R}$ is a Sierpiński space.

The topological product of two Sierpiński spaces is not, in general, a Sierpiński space. To see this, let us construct, under Martin's Axiom, a generalized Sierpinski subset $X$ of $\mathbf{R}$ with the property that

$$
X+X=\{y+z: y \in X, z \in X\}=\mathbf{R} .
$$

The construction of $X$ is very similar to the ones presented in [8] and [1]. Let $\alpha$ denote the smallest ordinal number of cardinality c. Let $\left\{x_{\xi}: \xi<\alpha\right\}$ be the family of all points of $\mathbf{R}$ and let $\left\{B_{\xi}: \xi<\alpha\right\}$ be the family of all Lebesgue measure zero Borel subsets of $\mathbf{R}$. Suppose that, for an ordinal $\zeta<\alpha$, two partial sequences

$$
\left\{y_{\xi}: \xi<\zeta\right\},\left\{z_{\xi}: \xi<\zeta\right\}
$$

of points of $\mathbf{R}$ have already been defined. Denote

$$
B_{\zeta}^{\prime}=\bigcup\left\{B_{\xi}: \xi<\zeta\right\}
$$

and take the point $x_{\zeta}$. Since $B_{\zeta}^{\prime}$ is of Lebesgue measure zero, we infer that the set

$$
\left(x_{\zeta}-\left(\mathbf{R} \backslash B_{\zeta}^{\prime}\right)\right) \cap\left(\mathbf{R} \backslash B_{\zeta}^{\prime}\right)
$$

is the complement of a Lebesgue measure zero set. Hence there are two distinct points $y_{\zeta}$ and $z_{\zeta}$ from $\mathbf{R} \backslash B_{\zeta}^{\prime}$ belonging to the set

$$
\mathbf{R} \backslash\left(\left\{y_{\xi}: \xi<\zeta\right\} \cup\left\{z_{\xi}: \xi<\zeta\right\}\right)
$$


and satisfying the relation

$$
x_{\zeta}=y_{\zeta}+z_{\zeta}
$$

We thus have defined the sequences

$$
\left\{y_{\xi}: \xi \leq \zeta\right\},\left\{z_{\xi}: \xi \leq \zeta\right\} .
$$

Proceeding in such a way, we finally obtain the $\alpha$-sequences

$$
\left\{y_{\xi}: \xi<\alpha\right\},\left\{z_{\xi}: \xi<\alpha\right\} .
$$

Now, putting

$$
X=\left\{y_{\xi}: \xi<\alpha\right\} \cup\left\{z_{\xi}: \xi<\alpha\right\},
$$

we get a generalized Sierpiński subset $X$ of $\mathbf{R}$ such that $X+X=\mathbf{R}$. The last property immediately implies that there exists a continuous mapping from the product space $X \times X$ onto $\mathbf{R}$. As mentioned above, under Martin's Axiom, $\mathbf{R}$ is not a Sierpiński space. Applying Theorem 2, we conclude that $X \times X$, being the product of two Sierpiński spaces, is not a Sierpiński space.

Let $E$ be a topological space which is not universal measure zero. A natural question arises: does there exist a Sierpiński subspace $E^{\prime}$ of $E$ with $\operatorname{card}\left(E^{\prime}\right)=\operatorname{card}(E)$ ? It is shown in [4] and [5] that the answer to this question is negative. Suppose now that $E$ is a non-universal measure zero, metrizable space whose weight is not real-valued measurable. Hence there exists a Borel diffused probability measure $\mu$ on $E$. This measure is concentrated on some separable subspace $F$ of $E$. Then, assuming Martin's Axiom and applying the construction similar to the one of a generalized Sierpiński subset of $\mathbf{R}$ (where the standard Lebesgue measure $\lambda$ plays the role of the restriction of $\mu$ to $F$ ), we come to a Sierpiński subspace $E^{\prime}$ of $F$ associated with $\mu$. In other words, $E^{\prime}$ is separable, $\operatorname{card}\left(E^{\prime}\right)=\mathbf{c}$ and, for any $\mu$-measure zero set $Z \subset E$, the relation

$$
\operatorname{card}\left(Z \cap E^{\prime}\right)<\mathbf{c}
$$

is satisfied. We thus conclude that, under Martin's Axiom, the answer to the question formulated above is positive for the class of all metrizable spaces of cardinality $\mathbf{c}$.

Assuming Martin's Axiom, we also have the following

Theorem 3. Let $E$ be a metrizable space of cardinality $\mathbf{c}$ equipped with a Borel diffused probability measure $\mu$, and let $X$ be a generalized Sierpiński subset of $E$ associated with $\mu$. Then, under Martin's Axiom, $X$ is of first category on each nonempty perfect subset of $E$, i.e. for any nonempty perfect set $P \subseteq E$, the intersection $X \cap P$ is of first category in $P$. 
Proof. Let $F$ denote a separable closed subset of $E$ for which $\mu(F)=1$ and $X \subset F$. Take an arbitrary nonempty perfect subset $P$ of $E$.

Suppose first that $\mu(P)=0$. Then, in view of the construction of $X$, we have $X \cap P \subset F$ and $\operatorname{card}(X \cap P)<\mathbf{c}$. But, under Martin's Axiom, any separable subset of $P$ with cardinality strictly less than $\mathbf{c}$ is of first category in $P$.

Suppose now that $\mu(P)>0$. Then, considering $\mu$ only on $P$ and applying to $P$ the existence of a separable support of $\mu$, we infer that there exists a partition $\{A, B\}$ of $P$ into two Borel sets, such that $\mu(A)=0$ and $B$ is of first category in $P$. Consequently, we have again $\operatorname{card}(X \cap A)<\mathbf{c}$ which implies that the separable set $X \cap A$ is of first category in $P$. Finally, we obtain that the set

$$
X \cap P=(X \cap A) \cup(X \cap B)
$$

is of first category in $P$, too, and the proof is complete.

Another proof of this statement, for a classical Sierpiński subset of $\mathbf{R}$, can be found in [7] and [8]. In addition, it is proved in [7] that, each Borel subset of a Sierpinski set is simultaneously $G_{\delta}$-set and $F_{\sigma}$-set. In other words, the Borel hierarchy in a Sierpiński set is almost trivial. So, it is not surprising the fact that (under the Continuum Hypothesis) the family of all analytic subsets of a Sierpiński set coincides with the family of all its Borel subsets. This statement remains true for any Sierpiński space having cardinality $\omega_{1}$ (note that such a space contains no uncountable discrete subspace; in particular, it satisfies the countable chain condition).

For a topological space $E$, let the symbol $A(E)$ denote the family of all analytic subsets of $E$. In other words, $A(E)$ consists of all those sets which can be obtained as a result of $(A)$-operation applied to an $(A)$-system of Borel subsets of $E$.

Theorem 4. Let $E$ be an arbitrary Sierpiński space of cardinality $\omega_{1}$. Then we have the equality

$$
A(E)=B(E) .
$$

Proof. Let $X$ be an analytic subset of $E$, i.e. $X$ is obtained as a result of $(A)$-operation applied to some $(A)$-system of Borel subsets of $E$. Suppose for a moment that $X$ is not Borel. Then, according to the classical theorem of Luzin and Sierpiński (cf. [7] or [8]), there exists a canonical representation of $X$ in the form

$$
X=\bigcup\left\{X_{\xi}: \xi<\omega_{1}\right\}
$$


where all $X_{\xi}$ are Borel subsets of $E$. Since $X$ is not Borel, there are uncountably many nonempty sets in the family

$$
\left(X_{\xi} \backslash \bigcup\left\{X_{\zeta}: \zeta<\xi\right\}\right)_{\xi<\omega_{1}} .
$$

Consequently, there exists an uncountable set $Y \subset X$ whose intersection with each set of the above-mentioned family is either empty or one-element. As demonstrated by Luzin and Sierpiński (see, e.g., [7] and [8]), the set $Y$ is universal measure zero (it should be noted here that their argument works in general situation, not only for Polish spaces). So we get a contradiction with our assumption that $E$ is a Sierpiński space. This contradiction shows us that all analytic subsets of $E$ must be Borel in $E$.

The classical construction of a Sierpiński set can be applied not only to $\mathbf{R}$ (or, more generally, to an uncountable Polish space equipped with a nonzero $\sigma$-finite Borel measure) but also to some other canonical spaces. Consider, for example, the commutative compact topological group $\Gamma=2^{\mathbf{c}}$ equipped with the Haar probability measure $\mu$. Let $I(\mu)$ denote the $\sigma$-ideal generated by the family of all $\mu$-measure zero subsets of $\Gamma$. It is well known that there exists a base of $I(\mu)$ whose cardinality equals c (see, e.g., [2] or [3]). Hence, by assuming the Continuum Hypothesis and utilizing the classical argument, it is not hard to construct in $\Gamma$ a Sierpiński set $X$ of cardinality c. The set $X$ will be a Sierpiński space in the sense of our definition. Moreover, it can easily be observed that $X$ is nonmeasurable with respect to the completion of $\mu$. Indeed, on the one hand, it is clear that $\mu^{*}(X)>0$. On the other hand, $\operatorname{since} \operatorname{card}(X)<\operatorname{card}(\Gamma)$, there are uncountably many pairwise disjoint translates of $X$ in $\Gamma$. So, taking into account the countable chain condition for $\mu$, we must have $\mu_{*}(X)=0$. Consequently, $X$ (and, moreover, each uncountable subset of $X$ ) is nonmeasurable with respect to the completion of $\mu$, and thus $X$ does not contain uncountable universal measure zero subspaces.

The same argument can be applied (under the Continuum Hypothesis) to the commutative compact topological group $\mathbf{S}^{\mathbf{c}}$ where $\mathbf{S}$ denotes the onedimensional torus. We recall that each of the above-mentioned spaces $2^{\mathbf{c}}$ and $\mathbf{S}^{\mathbf{c}}$ is nonmetrizable but separable.

\section{References}

[1] Cox, G.V., A universal null graph whose domain has positive measure, Colloq. Math. 46(2) (1982), 181-184.

[2] Halmos, P.R., Measure Theory, D. Van Nostrand, Princeton — New York, 1950.

[3] Hewitt, E., Ross, K., Abstract Harmonic Analysis, Vol 1, Springer-Verlag, Berlin, 1963. 
[4] Kharazishvili, A.B., Remark on Luzin sets and Sierpinski sets, Bull. Georgian Acad. Sci. 143(3) (1991), (in Russian).

[5] Kharazishvili, A.B., Generalized Sierpiński sets, Proc. Georgian Acad. Sci. Math. 1(5) (1993).

[6] Kunen, K., Set Theory, North-Holland, Amsterdam, 1980.

[7] Kuratowski, K., Topology, Vol 1, Academic Press, New York - London, 1966.

[8] Miller, A.W., Special subsets of the real line, in: "Handbook of Set-Theoretic Topology", North-Holland, Amsterdam, 1984.

[9] Morgan II, J.C., Point Set Theory, Marcel Dekker, Inc., New York — Basel, 1990.

[10] Oxtoby, J.C., Measure and Category, Springer-Verlag, Berlin, 1971.

\author{
A.B. KharazishVili \\ Institute of Applied Mathematics \\ of Tbilisi State University \\ UNIVERSITY STR. 2 \\ 380043 TBILISI 43 \\ Republic of Georgia
}

